\title{
Presencia de Cresta ósea en la Epífisis Proximal de la Tibia. Propuesta para su Incorporación en la Terminologia Anatomica
}

\author{
Presence of a Bony Crest in the Proximal Epiphysis of the Tibia. \\ Proposal for its Incorporation in the Terminologia Anatomica
}

Juan Pablo Pacheco Muñoz ; Rodrigo Alberto Lizama Pérez² \& Enrique Olave ${ }^{3}$

PACHECO, M. J. P.; LIZAMA, P. R. A. \& OLAVE, E. Presencia de cresta ósea en la epífisis proximal de la tibia. Propuesta para su incorporación en la Terminologia Anatomica. Int. J. Morphol., 39(2):560-563, 2021.

RESUMEN: La Terminologia Anatomica (TA) presenta las estructuras anatómicas en un lenguaje unificado para todas las estructuras del cuerpo humano. Sin embargo, hay características como algunos accidentes óseos que no se han considerado en las actualizaciones de la $T A$, ya sean epónimos que no se han relacionado con términos actuales o estructuras descritas clásicamente, como es el caso de una característica ósea que se observa y palpa fácilmente en la epífisis proximal de la tibia, a nivel del cóndilo lateral, uniendo el tubérculo anterolateral de la tibia con la tuberosidad de la misma. Esta característica corresponde a una elevación lineal, descrita como lugar de inserción del tracto iliotibial y del músculo tibial anterior. Basado en lo anterior, se analizaron 65 tibias de individuos Chilenos, adultos, de ambos sexos, 60 pertenecientes a la Universidad de La Frontera y 5 a la Universidad San Sebastián, considerando como criterio de inclusión la integridad del tejido compacto en la epífisis proximal. La revisión de las muestras reveló la presencia de una elevación lineal en el $100 \%$ de los casos, uniendo la tuberosidad de la tibia con el tubérculo anterolateral (Gerdy), no observándose elevación similar desde la tuberosidad de la tibia en dirección al cóndilo medial. Esta elevación es utilizada como referencia en la palpación durante la evaluación de la rodilla. Por las evidencias presentadas, consideramos que esta elevación debe considerarse en la $T A$ como una característica más de la tibia, en base a su disposición lineal y elevada, además de su dirección oblicua desde la tuberosidad de la tibia al tubérculo anterolateral de la misma, por lo que proponemos denominarla como cresta anterolateral de la tibia (Crista anterolateralis tibiae).

PALABRAS CLAVE: Terminologia Anatomica; Anatomía, Tibia, Cresta anterolateral, Tracto iliotibial, Músculo tibial anterior.

\section{INTRODUCCIÓN}

Las marcas óseas o accidentes de la superficie de un hueso son de amplio uso en anatomía humana. En las últimas décadas, en Terminologia Anatomica (TA) se han modificado, corregido, retirado y principalmente, incorporado nuevos términos (Vidal-Seguel et al., 2021). Por su parte, Vásquez \& del Sol (2020) constataron que, en la segunda edición de $T A$, los términos relacionados con el capítulo de osteología se han triplicado en número. Sin embargo, han reportado que no hay consenso en parámetros para incorporar o eliminar estructuras como las variaciones anatómicas. La literatura muestra algunos accidentes óseos que no se han incluido en $T A$ o cuyos epónimos no se han traducido a términos oficiales (Pacheco \& Lizama-Pérez, 2019). Uno de éstos, ausente en $T A$, corresponde a una elevación ubicada en la epífisis proximal de la tibia, descrita como una cresta oblicua con recorrido de proximal a distal que se extiende desde el tubérculo anterolateral de la tibia o tubérculo de Gerdy (Pacheco \& Lizama-Pérez) hasta el margen lateral de la tuberosidad de la tibia, señalándolo además como inserción del músculo tibial anterior y del tracto iliotibial (OrtsLlorca, 1959; Testut \& Latarjet, 1972; Bouchet \& Cuilleret, 1988; Rouvière \& Delmas, 2002).

Además, este accidente óseo se describe como referencia en procedimientos de anatomía palpatoria. Tixa (2006) la denomina como cresta oblicua de la tibia que une el tubérculo lateral de la tibia con el margen lateral de la tuberosidad de la misma, la cual es oblicua de arriba abajo y de atrás adelante, la que es directamente accesible y palpable por debajo de la piel. 
A pesar de la constatación y mención por parte de diferentes autores de la presencia de esta elevación, ésta aún no ha sido considerada por la TA. En este contexto, el objetivo de esta investigación fue identificar la presencia de ésta y su frecuencia, con la finalidad de buscar un respaldo que permitiera o no proponer su incorporación en la Terminologia Anatomica Internacional, siguiendo las indicaciones y sugerencias de la IFAA, la que señala que los nombres de las estructuras deben tener un valor informativo, estar escritos en latín como lengua base y no deben ser usados los epónimos.

\section{MATERIAL Y MÉTODO}

Se revisaron las tibias pertenecientes a la osteotecas de la Universidad de La Frontera y a la Universidad San Sebastián, Chile. Se incluyeron para su revisión sólo aquellas que mantenían indemne el tejido cortical e integridad de su epífisis proximal. Se obtuvo una muestra total de 65 tibias, 37 izquierdas y 28 derechas, de individuos chilenos, adultos, de ambos sexos, de las cuales 60 correspondían a la Universidad de La Frontera y 5 a la Universidad San Sebastián, sede Puerto Montt, Chile. Se identificaron el tubérculo anterolateral y la tuberosidad de la tibia para posteriormente verificar si entre éstas existía un accidente óseo que las uniera. Adicionalmente se observó si la tuberosidad de la tibia presentaba saliencias que la unieran medialmente con otras partes óseas, de tal forma de verificar o descartar una simetría de esta estructura.

\section{RESULTADOS}

El $100 \%$ de las muestras analizadas presentó una elevación ósea, lineal y oblicua, que unía la tuberosidad de la tibia con el tubérculo anterolateral, donde la característica común fue la continuidad de esta elevación y un margen definido a la observación y la palpación (Figs. 1a,b). No se encontró estructura similar en la zona medial de la epífisis proximal. Por sus características, este accidente óseo debe ser denominado como cresta anterolateral de la tibia (Crista anterolateralis tibiae).

\section{DISCUSIÓN}

El objetivo del presente estudio fue determinar la presencia de la cresta anterolateral de la tibia y su frecuencia de presentación, con la finalidad de buscar un respaldo
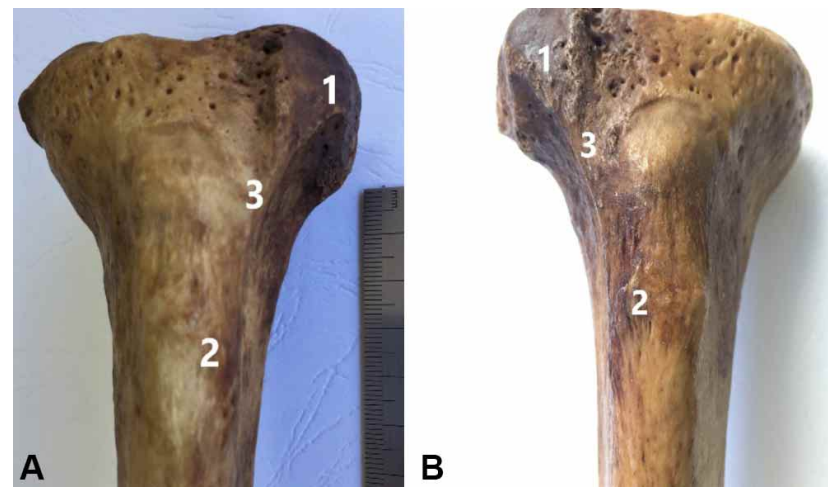

Fig. 1. (A) Tibia izquierda, (B) Tibia derecha: 1. Tubérculo anterolateral, 2. Tuberosidad de la tibia, 3. Cresta anterolateral.

que permita o no proponer su incorporación en la Terminologia Anatomica Internacional (Federative Committee on Anatomical Terminology, 1998). Esta estructura anatómica se encontró presente en la totalidad de las muestras estudiadas, sin embargo, no está incluida en la $T A$.

En la $T A$ se encuentran ausentes algunos términos correspondientes a estructuras anatómicas cuyos hallazgos no son recientes, lo cuales deberían estar incorporados (Fuentes et al., 2016) y la cresta anterolateral de la tibia es un ejemplo de ello.

Váquez \& del Sol indicaron que numerosos estudios han demostrado la presencia de estructuras anatómicas que constituyen variaciones y que, por su porcentaje, algunas de ellas debieran estar incluidas en $T A$ y otras no, constatando que, en la segunda edición de $T A$, los términos relacionados con el capítulo de osteología se han más que triplicado en número, concluyendo que la TA incorpora términos que constituyen variaciones anatómicas de muy baja frecuencia y no incluye términos anatómicos que por su mayor frecuencia deberían ser incorporados, ya sea como estructuras normales (fabela, músculo psoas menor).

En nuestro estudio la presentación de la cresta ósea fue regular en todas las muestras, lo que es un argumento importante para su inclusión como término oficial. En este caso, a diferencia de otras estructuras, este accidente óseo no corresponde a una variación anatómica, ya que de acuerdo a lo observado es parte de las características morfológicas constantes de la tibia.

Esta cresta no está incluida en la descripción de la tibia de algunos textos (García-Porrero \& Hurlé, 2005; Moore et al., 2010; Pró, 2014), sin embargo, textos clásicos de anatomía la han mencionado y descrito como lugar de inserción del tracto iliotibial y del músculo tibial anterior 
(Orts-Llorca; Rouvière \& Delmas) o como una elevación oblicua y rugosa que se desprende desde la tuberosidad de la tibia para terminar en el tubérculo anterolateral (Testut \& Latarjet; Bouchet \& Cuilleret).

Concordamos con Villarroel et al. (2020) cuando señalan que estructuras como éstas y particularmente en este caso, que se presentan exclusivamente en la zona lateral de la tibia, pueden ser un indicador de lateralidad en antropología o medicina forense.

Si bien la $T A$ se ha actualizado desde la creación de la Nomina Anatomica de Basilea, la versión moderna no es perfecta y puede ser criticada en el futuro por diversos autores, lo que es una buena práctica en Anatomía (Chmielewski \& Domagaa, 2020) Al respecto, la Anatomía como rama de las ciencias morfológicas, ha tratado de unificar criterios al momento de proponer nuevas estructuras o renombrar términos existentes en TA, para lo cual la Federación Internacional de Asociaciones de Anatomistas (IFAA) estableció una serie de indicaciones y sugerencias con la finalidad de regular y normalizar las denominaciones de las estructuras morfológicas entre las cuales, que todas se denominen con una sola palabra, que el lenguaje empleado sea el latín, que la estructura se describa lo más fiel a los principios de la geometría y la forma, que el término concentre la información y descripción de la estructura y que no se utilicen epónimos (Roa et al., 2016). En este contexto, concordamos que la corrección o incorporación de términos debe ser parte del trabajo de los anatomistas para mejorar la comunicación científica.

Siguiendo estos principios, basados en las características de la estructura descrita, proponemos incluir en $T A$ el término como Crista anterolateralis tibiae.

Esperamos que con la dinámica revisión de la $T A$, la presente propuesta sea discutida y aprobada por los integrantes del Programa Federativo de Terminología Anatómica (Federative International Programme for Anatomical Terminology, 2019), incorporando este término en futuras actualizaciones y publicaciones de la Terminologia Anatomica Internacional.

PACHECO, M. J. P.; LIZAMA, P. R. A. \& OLAVE, E. Presence of a bony crest in the proximal epiphysis of the tibia. Proposal for its incorporation in the Terminologia Anatomica. Int. J. Morphol.,39(2):560-563, 2021.

SUMMARY: Anatomical Terminology (AT) presents anatomical structures in a unified language for all structures of the human body. However, there are characteristics such as some bone accidents that have not been considered in the TA updates, whether they are eponyms that have not been related to current terms or classically described structures, as is the case of a characteristic bone that is easily observed and palpated in the proximal epiphysis of the tibia, at the level of the lateral condyle, joining the anterolateral tubercle of the tibia with the tuberosity of the tibia. This characteristic corresponds to a linear elevation, described as the insertion site of the iliotibial tract and the tibialis anterior muscle. Based on the above, 65 tibiae from Chilean individuals, adults, of both sexes, 60 belonging to the Universidad de La Frontera and 5 from the Universidad San Sebastián were analyzed, considering as an inclusion criterion the integrity of the compact tissue in the epiphysis proximal. The review of the samples revealed the presence of a linear elevation in $100 \%$ of the cases, joining the tibial tuberosity with the anterolateral tubercle (Gerdy), not observing similar elevation from the tibial tuberosity in the direction of the medial condyle. This elevation is used as a reference for palpation during knee evaluation. Based on the evidence presented, we consider that this elevation should be considered in TA as one more characteristic of the tibia, based on its linear and elevated disposition, in addition to its oblique direction from the tibial tuberosity to the anterolateral tubercle of the tibia, for what we propose to call it the Crista anterolateralis tibiae (anterolateral crest of the tibia).

KEY WORDS: Terminologia Anatomica; Anatomy; Tibia; Crista anterolateralis tibiae; Iliotibial tract; Tibialis anterior muscle.

\section{REFERENCIAS BIBLIOGRÁFICAS}

Bouchet, A. \& Cuilleret, J. Anatomía Descriptiva, Topográfica y Funcional. Buenos Aires, Médica Panamericana, 1988. Vol. 1.

Chmielewski, P. P. \& Domagaa, Z. A. Terminologia Anatomica and its practical usage: pitfalls and how to avoid them. Folia Morphol. (Warsz.), 79(2):198-204, 2020.

Federative Committee on Anatomical Terminology (FCAT). Terminologia Anatomica: International Anatomical Terminology. Stuttgart, Thieme, 1998.

Federative International Programme for Anatomical Terminology (FIPAT). Terminologia Anatomica. $2^{\mathrm{a}}$ ed. Halifax, FIPAT Library, Federative International Programme for Anatomical Terminology, 2019. Disponible en: https://fipat.library.dal.ca/TA2/

Fuentes, R.; Ottone, N. E.; Bucchi, C. \& Cantín, M. Analysis of terms used in the literature to refer to temporomandibular joint capsule and joint ligaments. Int. J. Morphol., 34(1):342-50, 2016.

García-Porrero, J. \& Hurlé, J. Anatomía Humana. Madrid, McGraw-Hill, 2005.

Moore, K. L.; Dalley, A. F. \& Agur, A. M. R. Anatomía con Orientación Clínica. $6^{\mathrm{a}}$ ed. Barcelona, Walters Kluwer-Lippincott Williams \& Wilkins, 2010.

Orts-Llorca, F. Anatomía Humana. $2^{\mathrm{a}}$ ed. Barcelona, Científico-Médica, 1959. Vol. 1.

Pacheco, J. P. \& Lizama-Pérez, R. Gerdy's tubercle. A forgotten structure in the International Anatomical Terminology. Int. J. Morphol., 37(4):1305-9, 2019

Pró, E. A. Anatomía Clínica. $2^{\text {a }}$ ed. Buenos Aires, Médica Panamericana, 2014.

Roa, I.; Vásquez, B. \& Contreras, M. Eponyms persistence in Terminologia Histologica. Int. J. Morphol., 34(4):1245-52, 2016. 
Rouvière, H. \& Delmas, A. Anatomía humana: descriptiva, topográfica y funcional. 10 ${ }^{\mathrm{a}}$ ed. Barcelona, Masson, 2002. Vol. 3.

Testut, L. \& Latarjet, A. Tratado de Anatomía Humana. $9^{\mathrm{a}}$ ed. Barcelona, Salvat, 1972. Vol. 1.

Tixa, S. Atlas de Anatomía Palpatoria. $3^{\mathrm{a}}$ ed. Barcelona, Elsevier-Masson, 2006. Vol. 2.

Vásquez, B. \& del Sol, M. What is the Critera Used to Include Certain Anatomical Variations in Terminologia Anatomica? Int. J. Morphol., 38(4):1136-41, 2020.

Vidal-Seguel, N.; Miranda-Krause, R. E.; Pumeyrau-Solar, M. \& Vásquez, B. Definitions of the landmarks, bony marks or accidentes of the bone surface in human anatomy. Int. J. Morphol., 39(1):335-40, 2021.

Villarroel, M.; Villagrán, F.; Olave, E. \& Riveros, A. Spiral line of the Femur. Int. J. Morphol., 38(1):193-8, 2020.
Dirección de correspondencia:

Prof. Mg. Juan Pablo Pacheco Muñoz

Universidad San Sebastián

Lago Panguipulli 1390

Pichi Pelluco

Puerto Montt

CHILE

Email: juan.pacheco@uss.cl

Recibido: 16-01-2021

Aceptado:17-02-2021 